\title{
Relaxation of Electron Spin during High-Field Transport in GaAs Bulk
}

\author{
S. Spezia ${ }^{a}$, D. Persano Adorno ${ }^{a}$, N. Pizzolato ${ }^{a b}$, B. Spagnolo ${ }^{a b}$ \\ ${ }^{a}$ Dipartimento di Fisica e Tecnologie Relative, \\ Università di Palermo and CNISM, \\ Viale delle Scienze, edificio 18, I-90128 Palermo, Italy \\ ${ }^{b}$ Group of Interdisciplinary Physics \\ E-mail: stefano.spezia@gmail.com
}

\begin{abstract}
A semiclassical Monte Carlo approach is adopted to study the multivalley spin depolarization of drifting electrons in a doped n-type GaAs bulk semiconductor, in a wide range of lattice temperature $\left(40<T_{L}<300 \mathrm{~K}\right)$ and doping density $\left(10^{13}<n<10^{16} \mathrm{~cm}^{-3}\right)$. The decay of the initial non-equilibrium spin polarization of the conduction electrons is investigated as a function of the amplitude of the driving static electric field, ranging between 0.1 and $6 \mathrm{kV} / \mathrm{cm}$, by considering the spin dynamics of electrons in both the $\Gamma$ and the upper valleys of the semiconductor. Doping density considerably affects spin relaxation at low temperature and weak intensity of the driving electric field. At high values of the electric field, the strong spin-orbit coupling of electrons in the $L$-valleys significantly reduces the average spin polarization lifetime, but, unexpectedly, for field amplitudes greater than $2.5 \mathrm{kV} / \mathrm{cm}$, the spin lifetime increases with the lattice temperature. Our numerical findings are validated by a good agreement with the available experimental results and with calculations recently obtained by a different theoretical approach.
\end{abstract}

PACS numbers: 72.25.Dc,72.25.Rb,72.20.Ht,02.50.Ng 


\section{Introduction}

The processing of a high volume of information and world wide communication is, at the present, based on semiconductor technology, whereas information storage devices rely on multilayers of magnetic metals and insulators. Semiconductor spintronics offers a possible direction of technological research towards the development of hybrid devices that could perform logic operations, communication and storage, within the same material technology: information could be stored in a system of polarized electron

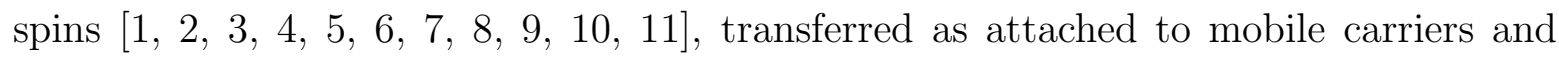
finally detected. The possibility of obtaining long spin relaxation times or spin diffusion lengths in electronic materials makes spintronics a viable prospective technology. Nevertheless, the designers of spin devices have to worry about the loss of spin polarization (spin coherence) before, during and after the necessary manipulations. In particular, efficient injection, transport, control and detection of spin polarization must be carefully treated [2]. Electron-spin states depolarize by scattering with imperfections or elementary excitations of the medium, such as phonons. Furthermore, miniaturization process brings the system to experience very intense electric fields, even when the applied voltages are very low. This means that, for the operability of prospective spintronic devices, the features of spin relaxation at relatively high electric fields should be firstly understood. In recent years there was a proliferation of experimental works in which the influence of transport conditions on relaxation of spins in semiconductors has been investigated [12, 13, 14, 15, 16, 17, 18]. All these works are focused on the study of coherent spin transport at low temperatures $\left(T_{L}<30 \mathrm{~K}\right)$ and under the influence of weak electric fields $(F<0.1 \mathrm{kV} / \mathrm{cm})$, except for few works [12, 13] in which spin depolarization has been investigated with driving fields up to $6 \mathrm{kV} / \mathrm{cm}$.

The temporal evolution of the spin and the evolution of the momentum of an electron cannot be separated. The spin depolarization rates are functionals of the electron distribution function in momentum space which continuously evolves with time when an electric field is applied to drive the transport. Thus, the dephasing rate is a dynamic variable that needs to be treated self-consistently in step with the dynamic evolution of the electron's momentum. A way to solve this problem is to describe the transport of spin polarization by making use of Boltzmann-like kinetic equations. This can be done within the density matrix approach [19], methods of nonequilibrium Green's functions, as the microscopic kinetic spin Bloch equation approach [20, 21, 22, 23, 24, 11, or Wigner functions [25, 26], where spin property is accounted for starting from quantum mechanics equations.

Another way is to use a semiclassical Monte Carlo approach, by taking into account the spin polarization dynamics with the inclusion in the code of the precession mechanism of the spin polarization vector [27, 28, 29, 30, 31, 32, 33, 34].

Both methods allow to include the relevant spin relaxation phenomena for electron systems and take into account the details of electron scattering mechanisms, material properties and specific device design; their predictions have been demonstrated to be in 
good agreement with experiments.

Theoretical descriptions of the transport of spin-polarized electrons have been also achieved by the drift-diffusion approximation. The existing drift-diffusion schemes can be classified into two approaches accounting the spin degree of freedom differently: the two-component drift-diffusion model and the density-matrix based approximations [35, 36, 37]. General conditions for the applicability of these approximations are not different from the usual conditions of applicability of drift-diffusion approximations.

Despite decades of studies, most of theoretical or simulative works have considered only the central valley $\Gamma$ since the spin-orbit coupling parameters of the upper conduction bands have been only recently theoretically calculated by $\mathrm{Fu}$ et al. [38. Monte Carlo approaches have been widely adopted by groups of scientists to study spin polarized transport in 2D channels, heterostructures, quantum wells, quantum wires [27, 28, 30, 31, 32, 33. However, till today, to the best of our knowledge, in semiconductor bulk structures a theoretical investigation of the influence of transport conditions on the spin depolarization in the presence of high electric fields, comprehensive of the effects of both lattice temperature and impurity density, is still lacking.

Aim of this work is to numerically estimate the spin lifetimes of an ensemble of initially-polarized electrons drifting in doped n-type GaAs bulks, with the lattice temperature, doping density and electric field amplitude ranging in a wide interval of values, focusing on the effects due to the inclusion of upper valleys of the semiconductor on the mechanism of depolarization.

The paper is organized as follows: in Sec. 2 we briefly describe the multivalley model, used for the study of the spin depolarization dynamics and the Monte Carlo simulator; in Sec. 3 the numerical results are given and discussed. Final comments and conclusions are given in Sec. 4 .

\section{Theory and Monte Carlo approach}

\subsection{Spin dynamics and multivalley model}

Spin dephasing may be caused by interactions with local magnetic fields originating from nuclei and spin-orbit interactions or magnetic impurities. The most relevant spin relaxation mechanisms for an electron system under non degenerate regime are: (i) the Elliott-Yafet (EY) mechanism, in which electron spins have a small chance to flip during each scattering, due to the spin mixing in the conduction band [39, 40]; (ii) the Dyakonov-Perel (DP) mechanism, based on the spin-orbit splitting of the conduction band in non-centrosymmetric semiconductors, in which the electron spins decay due to their precession around the $\mathbf{k}$-dependent spin-orbit fields (inhomogeneous broadening) during the free flight between two successive scattering events [41, 42, 7]; (iii) the BirAronov-Pikus (BAP) mechanism, in which electrons exchange their spins with holes [43. Hyperfine interaction is another mechanism, usually important for spin relaxation of localized electrons, but ineffective in metallic regime where most of the carriers are 
in extended states [44, 45, 46].

Previous theoretical [24, 11] and experimental [47] investigations indicate that the the EY mechanism is totally irrelevant on electron spin relaxation in n-type III-V semiconductors. Hence, in this work we analyze the spin depolarization of drifting electrons in n-type GaAs semiconductors by considering only the D'yakonov-Perel process.

By following the semiclassical formalism, the term of the single electron Hamiltonian which accounts for the spin-orbit interaction can be written as

$$
H_{S O}=\frac{\hbar}{2} \vec{\sigma} \cdot \vec{\Omega} \text {. }
$$

It represents the energy of electron spins precessing around an effective magnetic field $\left[\vec{B}=\hbar \vec{\Omega} / \mu_{B} g\right]$ with angular frequency $\vec{\Omega}$, which depends on the orientation of the electron momentum vector with respect to the crystal axes. Near the bottom of each valley, the precession vector can be written as [46, 38]

$$
\vec{\Omega}_{\Gamma}=\beta_{\Gamma}\left[k_{x}\left(k_{y}^{2}-k_{z}^{2}\right) \hat{x}+k_{y}\left(k_{z}^{2}-k_{x}^{2}\right) \hat{y}+k_{z}\left(k_{x}^{2}-k_{y}^{2}\right) \hat{z}\right]
$$

in the $\Gamma$-valley, and

$$
\vec{\Omega}_{L}=\frac{\beta_{L}}{\sqrt{3}}\left[\hat{x}\left(k_{y}-k_{z}\right)+\hat{y}\left(k_{z}-k_{x}\right)+\hat{z}\left(k_{x}-k_{y}\right)\right]
$$

in the L-valleys, located along the [111] direction of the crystallographic axes. In equations (2)-(3),$k_{i}(i=x, y, z)$ are the components of the electron wave vector. $\beta_{\Gamma}$ and $\beta_{L}$ are the spin-orbit coupling coefficients, crucial parameters for the simulation of spin polarization. Here, we assume $\beta_{L}=0.26 \mathrm{eV} / \AA \cdot 2 / \hbar$, as recently theoretically estimated [38. In $\Gamma$-valley we consider the effects of nonparabolicity on the spin-orbit splitting by using [46],

$$
\beta_{\Gamma}=\frac{\alpha \hbar^{2}}{m \sqrt{2 m E_{g}}}\left(1-\frac{E(\vec{k})}{E_{g}} \frac{9-7 \eta+2 \eta^{2}}{3-\eta}\right)
$$

where $\alpha=0.029$ is a dimensionless material-specific parameter, $\eta=\Delta /\left(E_{g}+\Delta\right)$, with $\Delta=0.341 \mathrm{eV}$ the spin-orbit splitting of the valence band, $E_{g}$ is the energy separation between the conduction band and valence band at the $\Gamma$ point, $m$ the effective mass and $E(\vec{k})$ the electron energy.

The quantum-mechanical description of electron spin evolution is equivalent to that of the classical momentum $\vec{S}$ experiencing the effective magnetic field, as described by the equation of motion

$$
\frac{d \vec{S}}{d t}=\vec{\Omega} \times \vec{S}
$$

Every scattering event changes the orientation of the effective magnetic field $\vec{B}$ (that strongly depends on $\vec{k}$ ) and the direction of the spin precession axis. 


\subsection{The Monte Carlo algorithm}

The Monte Carlo approach is one of the most powerful methods to simulate the transport properties in semiconductor devices beyond the quasi-equilibrium approximations [48, 49, 50]. In fact, owing to its flexibility, the Monte Carlo method presents the remarkable advantage of giving a detailed description of the particle motion in the semiconductor by taking into account the details of collisions with impurities, phonons, etc., specific device design and material parameters, and allows us to obtain all the needed information, such as the average velocity of electrons, temperature, current density, etc., directly without the need of calculating first the electron distribution function. In simulations, between two successive scattering events, each electron propagates along a classical trajectory and, according to the classical equations of motion, it is affected by the presence of external fields. The time interval between two collisions (time of free flight), the scattering mechanisms, the collisional angle, and all the parameters of the problem are chosen in a stochastic way, making a mapping between the probability density of the given microscopic process and a uniform distribution of random numbers.

In our code the conduction bands of GaAs are represented by the $\Gamma$-valley and by four equivalent $\mathrm{L}$ valleys. We do not consider the $X$-valleys because, even for the highest value of the driving field $(F=6 \mathrm{kV} / \mathrm{cm})$, the percentage of electrons in these valleys is always lower than $0.1 \%$. The algorithm includes: (i) the intravalley scattering with acoustic phonons, ionized impurities, acoustic piezoelectric phonons, polar optical phonons, and for the the L-valleys also the scattering with optical nonpolar phonons; (ii) the intervalley scattering with the optical nonpolar phonons among the two valleys. The complete set of n-type GaAs parameters used in our calculations is listed in Ref. [51]. The scattering probabilities are calculated by the Fermi Golden Rule and assumed to be both field and spin independent; accordingly, the influence of the external fields is only indirect through the field-modified electron velocities. Nonlinear interactions of the field with the lattice and bound carriers are neglected. We also neglect electron-electron interactions and consider electrons to be independent [27]. The spin polarization vector is included into the Monte Carlo algorithm and calculated for each free carrier. From Eq. (5), the Monte Carlo (MC) simulator calculates the electron spin precession, by taking into account the scattering-induced deviations of precession vector suffered after each collision.

All simulations are performed by using a temporal step of $10 \mathrm{fs}$ and an ensemble of $5 \cdot 10^{4}$ electrons to collect spin statistics. We assume that all donors are ionized and that the free electron concentration is equal to the doping concentration $n$. 


\section{Numerical Results}

\subsection{Spin lifetime calculation}

The initial non-equilibrium spin polarization decays with time as the electrons, driven by a static electric field, move through the medium, experiencing elastic and anelastic collisions. Since scattering events randomize the direction of $\vec{\Omega}$, during the motion, the polarization vector of the electron spin experiences a slow angular diffusion. The dephasing of each individual electron spin produces a distribution of spin states that results in an effective depolarization, which is calculated by ensemble-averaging over the spin of all the electrons.

The simulation of the spin relaxation starts with all the electrons of the ensemble initially polarized $(\langle\vec{S}\rangle=1)$ along the $\hat{x}$-axis at the injection plane $\left(x_{0}=0\right)$. After a transient time of typically $10^{4}$ time steps, long enough to achieve the steady-state transport regime, the electron spins are initialized, the spin relaxation begins and the quantity $\langle\vec{S}\rangle$ is calculated as a function of time. In order to extract the characteristic time $\tau$ of the spin relaxation, the obtained trend of the spin dephasing is fitted by the following exponentially time decaying law

$$
\left\langle S_{x}\right\rangle(t)=A \cdot \exp (-t / \tau)
$$

with $A$ a normalization factor.

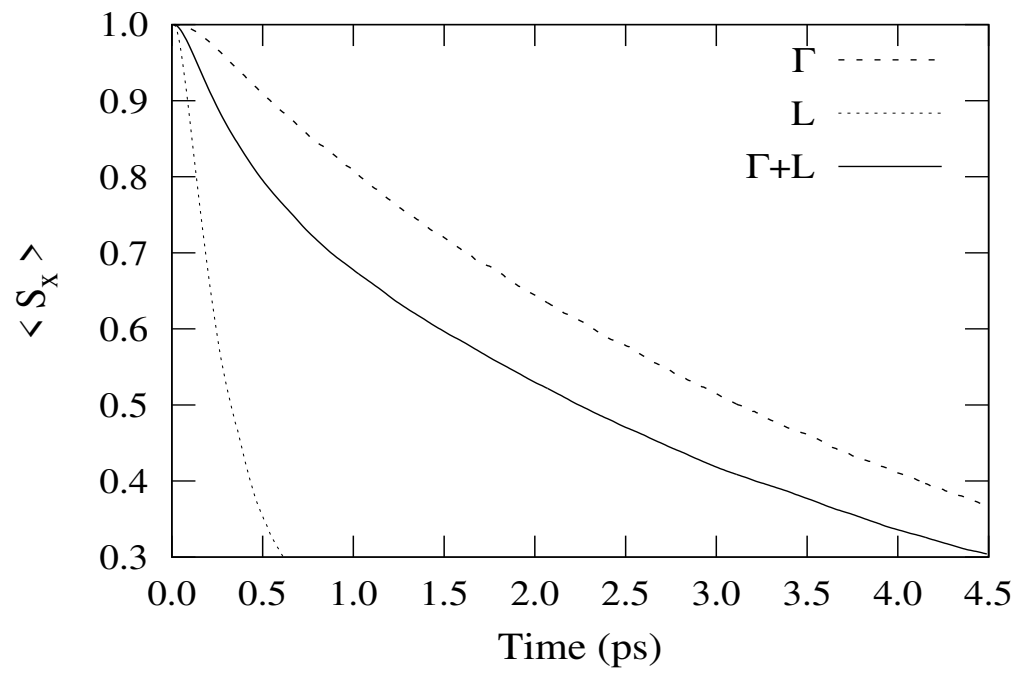

Figure 1. Average electron spin polarization $\left\langle S_{x}\right\rangle$ as a function of time, by only considering the electrons drifting into the $\Gamma$-valley (dashed line), into the $L$-valleys (dotted line) and into the $(\Gamma+L)$-valleys (solid line). $F=5 \mathrm{kV} / \mathrm{cm}, n=10^{13} \mathrm{~cm}^{-3}$ and $T_{L}=300 \mathrm{~K}$.

In Fig. 1 we show the average electron spin polarization $\left\langle S_{x}\right\rangle$, in the presence of a driving electric field, with amplitude $F=5 \mathrm{kV} / \mathrm{cm}$ and directed along the $\hat{x}$-axis, with density $n=10^{13} \mathrm{~cm}^{-3}$ and lattice temperature $T_{L}=300 \mathrm{~K}$. This value of field amplitude is high enough to allow almost $21 \%$ of all electrons to visit the $L$ valleys. The 


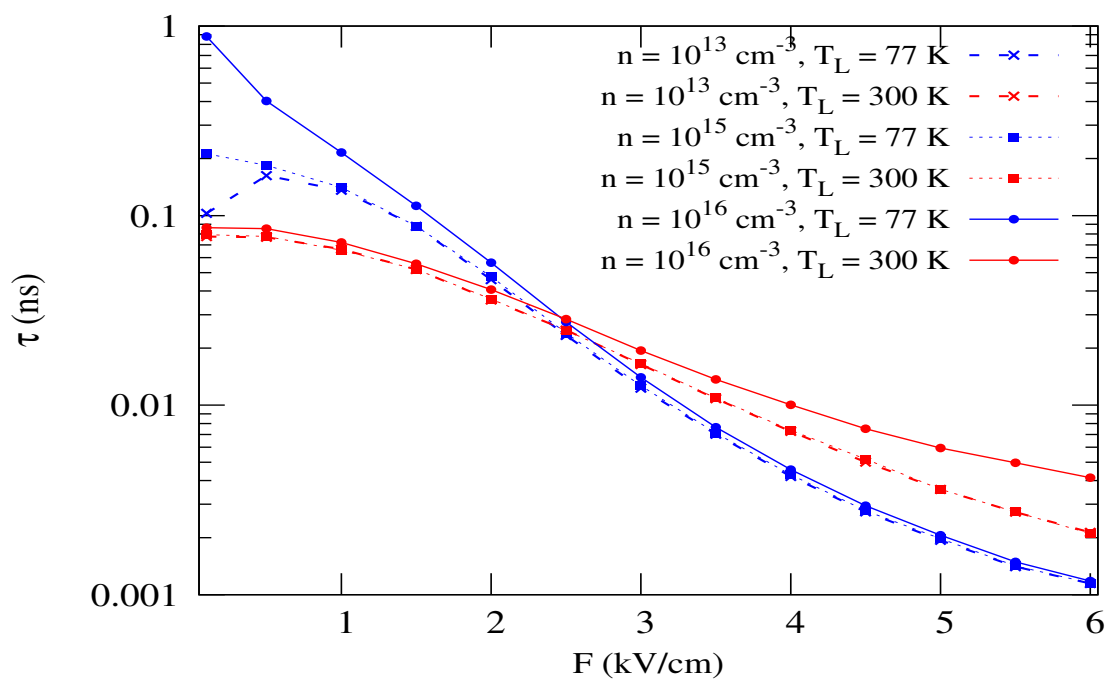

Figure 2. Spin lifetime $\tau$ as a function of the electric field amplitude $F$, at $T_{L}=77$ $\mathrm{K}$ (blue lines) and $T_{L}=300 \mathrm{~K}$ (red lines), for three values of doping density, namely $n=10^{13} \mathrm{~cm}^{-3}, n=10^{15} \mathrm{~cm}^{-3}$ and $n=10^{16} \mathrm{~cm}^{-3}$, with the electrons drifting in both the $\Gamma$ and the $L$-valleys.
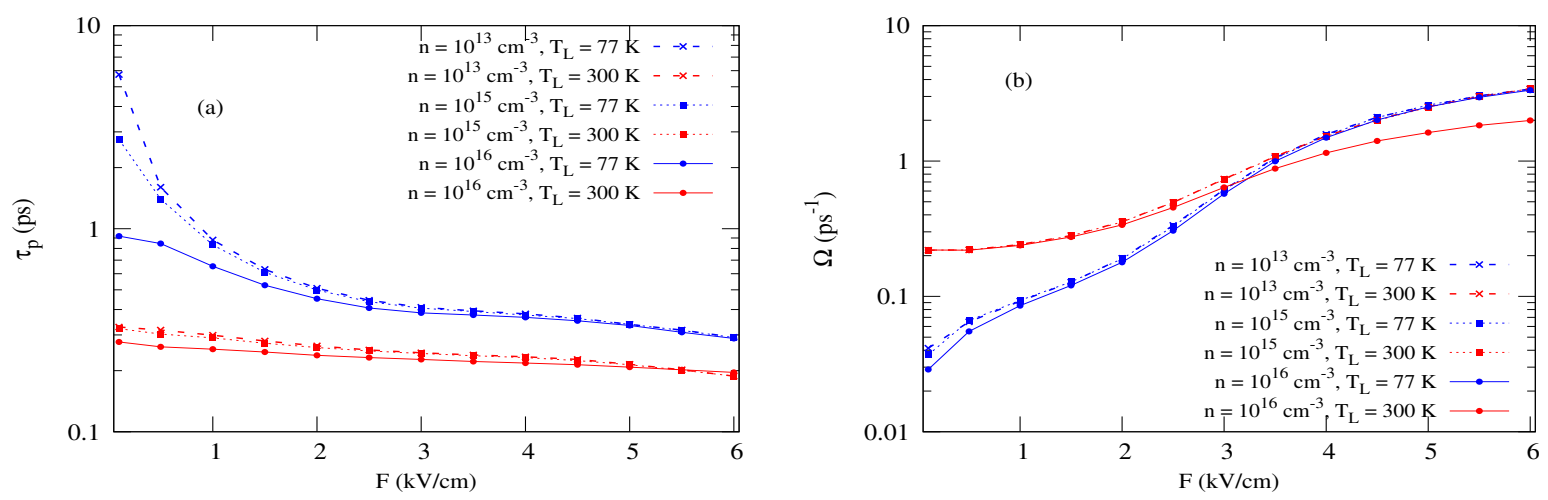

Figure 3. (a) Momentum scattering time $\tau_{p}$ and (b) spin precession frequency $\Omega$ as a function of the electric field amplitude $F$, at $T_{L}=77 \mathrm{~K}$ (blue lines) and $T_{L}=300 \mathrm{~K}$ (red lines), for three values of doping density, namely $n=10^{13}, 10^{15}$ and $10^{16} \mathrm{~cm}^{-3}$.

curves represent the decreasing trend of $\left\langle S_{x}\right\rangle$ vs. time by firstly considering only the electrons drifting into the $\Gamma$-valley (dashed line), secondly, by solely taking into account the electrons moving into the $L$-valleys (dotted line) and, finally, by considering the electrons moving into both the $\Gamma$ and the $L$-valleys (solid line). We find a significant reduction of the average spin polarization lifetime caused by the spin-orbit coupling in $L$-valleys stronger with respect to that in $\Gamma$-valley, according to the theoretical results obtained by Zhang et al. [23] on quantum wells. The transition of about $21 \%$ of electrons to the $L$-valleys leads to an increase of efficacy of the dephasing mechanism, which brings to a reduction of $\left\langle S_{x}\right\rangle$ over time in the range $15 \div 20 \%$ 


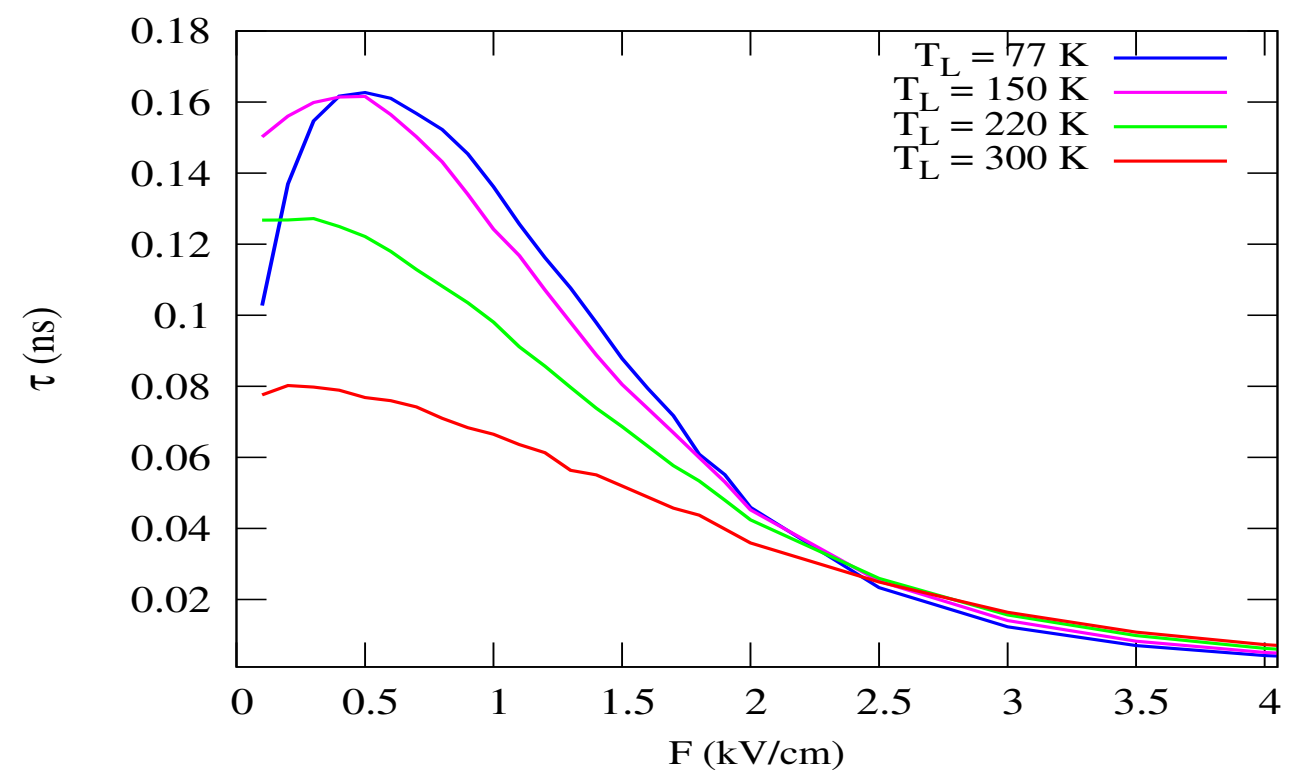

Figure 4. Spin lifetime $\tau$ as a function of the electric field amplitude $F$, at $n=10^{13}$ $\mathrm{cm}^{-3}$ for different values of lattice temperature, namely $T_{L}=77,150,220,300 \mathrm{~K}$, with electrons drifting in both the $\Gamma$ and the $L$-valleys.

\subsection{Effects of temperature and doping density on spin relaxation}

In Fig. 2, we show the spin depolarization time $\tau$ as a function of the electric field amplitude $F$, for two values of the lattice temperature, namely $T_{L}=77$ (blue curves) and $300 \mathrm{~K}$ (red curves) and three values of doping density $n=10^{13}, 10^{15}$ and $10^{16}$ $\mathrm{cm}^{-3}$, respectively, leaving the electrons free to drift in both the $\Gamma$ and the $L$-valleys. Except for the case at the doping density $n=10^{13} \mathrm{~cm}^{-3}$ and $T_{L}=77 \mathrm{~K}$, we find that $\tau$ is always a monotonic decreasing function of $F$. In fact, when the field amplitude becomes larger, the electron momentum $\mathbf{k}$ increases, causing a stronger effective magnetic field, as expected by Equations (2)-(3) . Consequently, the electron precession frequency becomes higher, inducing a faster spin relaxation [41.

For field amplitudes greater than $2.5 \mathrm{kV} / \mathrm{cm}$, at $T_{L}=300 \mathrm{~K}$ we find depolarization times longer than those obtained at $T_{L}=77 \mathrm{~K}$. In order to avoid that the observed behaviour could be ascribed only to stochastic fluctuations of MC computations, we have calculated the statistical error associated to our simulated data. We have repeated our simulations ten times, finding a maximum spread of $0.05 \mathrm{ps}$, which corresponds to about $1 \%$ of the observed variation of $\tau$ with the temperature.

We have investigated the counterintuitive behavior of longer average spin lifetimes obtained for hotter electrons, by adopting the proportionality law of Perel [41]:

$$
\frac{1}{\tau} \propto \Omega^{2} \tau_{p}
$$

where $\Omega$ is the spin precession frequency and $\tau_{p}$ the momentum characteristic relaxation time, corresponding to the time scale of the scattering events. We have calculated the spin precession frequency and the momentum characteristic scattering time for each 
electron of our ensemble and in Fig. 3, we show the average momentum scattering time $\tau_{p}$ (panel (a)) and the average spin precession frequency $\Omega$ (panel (b)) as a function of the electric field amplitude $F$, at $T_{L}=77$ and $300 \mathrm{~K}$, for the three values of doping density $n=10^{13}, 10^{15}$ and $10^{16} \mathrm{~cm}^{-3}$.

The panel (a) of Fig. 3 shows that $\tau_{p}$ is a monotonically decreasing function of $F$ for every values of $n$ and $T_{L}$. At the higher temperature $\left(T_{L}=300 \mathrm{~K}\right)$ we find low values of $\tau_{p}$, because electrons experience a greater number of scattering events, both in the $\Gamma$ valley and in $L$-valleys. Moreover, the curves at room temperature are characterized by only a slight slope, because in this case the thermal energy of the electrons is dominant with respect to the drift kinetic energy. At $T_{L}=77 \mathrm{~K}$ and for very low values of the electric field amplitude, since the scattering events are mainly due to ionized impurities, $\tau_{p}$ is greatly dependent on $n$, increasing its value at lower densities. At $T_{L}=300 \mathrm{~K}, \tau_{p}$ is nearly independent on the doping density since the dominant scattering mechanism is due to the optical phonons.

The panel (b) of Fig. 3 shows that the spin precession frequency $\Omega$ is an increasing monotonic function of $F$. For $F<3 \mathrm{kV} / \mathrm{cm}$, independently from the values of the doping density, the values of $\Omega$ obtained at room temperature are larger than those obtained at $T_{L}=77 \mathrm{~K}$. The increase of the spin precession frequency for electrons moving at higher temperatures is explained by the increasing number of electron transitions from the $\Gamma$ to $L$ valleys, being the value of the spin-orbit coupling coefficient in the $L$-valleys one order of magnitude greater than that of $\Gamma$-valley. At $T_{L}=77 \mathrm{~K}$, for $F<3$ $\mathrm{kV} / \mathrm{cm}$, the percentage of electrons in the central valley $\Gamma$ is practically unitary and the spin precession frequency increases as the third power of electron momentum $\vec{k}$, which increases with $F$ according to the Eq. (2). When $F$ is greater than $3 \mathrm{kV} / \mathrm{cm}$, the percentage of electrons in the L-valley is high enough to lead $\Omega$ for having a nearly linear trend (see Eq. (3) ). At $T_{L}=300 \mathrm{~K}$, for $F<3 \mathrm{kV} / \mathrm{cm}$, the term of thermal energy is dominant with respect to the drift kinetic energy and $\Omega$ vs. $F$ shows a more slight increase.

For $F>3 \mathrm{kV} / \mathrm{cm}$, independently from the values of $T_{L}$ and $n$, the action of $F$ wins on the disorder due to the lattice temperature. In fact, except for the data obtained at $T_{L}=300 \mathrm{~K}$ and $n=10^{16} \mathrm{~cm}^{-3}$, which show lower values of $\Omega$, all curves coincide. The detached curve $T_{L}=300 \mathrm{~K}$ and $n=10^{16} \mathrm{~cm}^{-3}$ is ascribed to a strong reduction of the percentage of electrons present in the L-valleys.

The calculation of the square of the spin precession frequency times the momentum relaxation time as a function of the electron energy for each electron of the ensemble shows that for $F>2.5 \mathrm{kV} / \mathrm{cm}$ the average value of $\Omega^{2} \tau_{p}$ obtained at $T_{L}=77 \mathrm{~K}$ is greater than that at $T_{L}=300 \mathrm{~K}$. This finding explains the longer lifetimes observed at higher temperatures for field amplitudes greater than $2.5 \mathrm{kV} / \mathrm{cm}$.

To highlight on the nonmonotonic electric field dependence of $\tau$, observed at the doping density $n=10^{13} \mathrm{~cm}^{-3}$, we have investigated the spin lifetime dependence on $F$ also for different values of the lattice temperature, namely $T_{L}=77,150,220,300 \mathrm{~K}$ (see Fig. 4). Up to $T_{L}=150 \mathrm{~K}$ the decoherence times slightly depend on the temperature 
and are characterized by a marked maximum. The presence of a maximum in the spin depolarization time can be explained by the interplay between two competing factors, both due to the increase of the electric field. In the momentum space, at greater field amplitudes, the electrons occupy states with larger $\vec{k}$, characterized by a stronger spinorbit coupling, causing an enhancement of the spin inhomogeneous broadening. On the other hand, a larger electric field also brings about an increase of the number of scattering events, giving rise to a reduction of the momentum relaxation time. This in turn causes an increase of the spin relaxation time as follows from Eq. 7 .

At low values of temperature and for electric field amplitudes $0.1 \leq F \leq 0.5 \mathrm{kV} / \mathrm{cm}$ the inhomogeneous broadening is still marginal and the spin relaxation phenomenon is dominated by the momentum scattering. In particular, the number of electron scattering events, which are mainly due to interactions with acoustic phonons at very weak electric fields, increases its value because of the triggering of the scattering mechanism by ionized impurities, causing a reduction of $\tau_{p}$. For field amplitudes greater than $\approx 0.5 \mathrm{kV} / \mathrm{cm}$, the enhancement of the spin-orbit coupling, which is k-cubic dependent, is faster than

the decrease on $\tau_{p}$. Consequently, the spin lifetime starts to decrease with the increasing of the electric field. The nonmonotonic electric field dependence of $\tau$ is not observed for $T_{L}>150 \mathrm{~K}$ where, because of the greater drift electron velocities, the loss of spin polarization is mainly due to the strong effective magnetic field.

\subsection{Comparison with experiments and with other theoretical approaches}

Unfortunately, till today experimental investigations of the ultrafast relaxation of electron spin during drift transport in bulk semiconductors, at both sample temperatures higher than $30 \mathrm{~K}$ and applied field amplitudes greater than $0.1 \mathrm{kV} / \mathrm{cm}$, are still missing.

Although the main aim of this work is the investigation of the influence of transport conditions on the spin relaxation of electrons driven by high-intensity electric fields, including the effects of choosing both the lattice temperature and impurity density in a wide range of values, in order to validate the prediction capability of our MC code, we have performed a comparison between our numerical spin relaxation times and very recent experimental results on the electron-spin-relaxation rate, obtained by Ref. [52]. These experiments were carried out by performing spin noise spectroscopy on a sample of n-type GaAs at a doping concentration of $n=2.7 \cdot 10^{15} \mathrm{~cm}^{-3}$, without any driving field and for lattice temperatures $T_{L}$ between 4 and $80 \mathrm{~K}$. In Fig. 5 we plot the temperature dependence of the spin-relaxation rate calculated from our code (solid line), together with the experimental data (circles, sample B in Ref. [52]). In order to best fit the experimental points with our numerical trend, we have utilized the spin-orbit coupling coefficient in $\Gamma$-valley $\beta_{\Gamma}$ as a free parameter, obtaining the best agreement with $\beta_{\Gamma}=19 \mathrm{eV} \cdot \AA^{3}$. This value is only slightly different from the value $\left(23.8 \mathrm{eV} \cdot \AA^{3}\right)$, recently estimated by using the tight binding theory [38]. However this value is still within the reasonable range of values calculated and measured via various methods, as reported in Ref. [53]. For sample temperatures greater than $45 \mathrm{~K}$, our numerical trend well agrees 
with the experimental data, while at lower temperatures a considerable discrepancy is found, probably due to the neglecting the electron-electron scattering mechanism. In fact, as shown in Ref. [24] at $T_{L}=40 \mathrm{~K}$, the assumption of neglecting the Coulomb term gives spin lifetime values smaller (hence relaxation rates higher) than those obtained from full calculation including all scattering mechanisms.

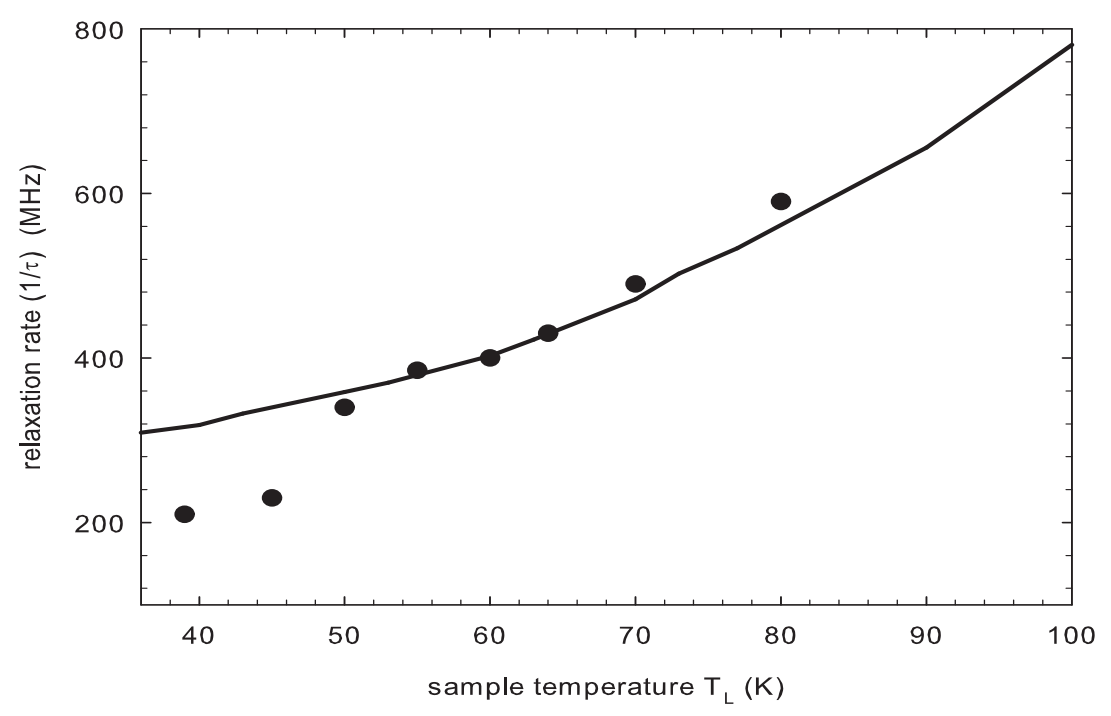

Figure 5. Temperature depended measurements of the spin-relaxation rate from the experiment (sample B) in Ref. [52] (circles) and numerical data obtained from our Monte Carlo code (solid curve), $n=2.7 \cdot 10^{15} \mathrm{~cm}^{-3}, \beta_{\Gamma}=19 \mathrm{eV} \cdot \AA^{3}$

In order to further test the effectiveness of our code we have compared our onevalley numerical data with the calculation of the effects of a low-amplitude electric field $(F \leq 2 \mathrm{kV} / \mathrm{cm})$ on spin relaxation in n-type III-V semiconductor bulks, lately obtained from the fully microscopic kinetic spin Bloch equation (KSBE) approach [24]. To the best of our knowledge, that paper is the only one in which the electric field dependence of spin lifetime has been investigated. In Fig. 6 we plot the ratio of the spin relaxation time under electric field to the electric-field-free one $\tau(F) / \tau(F=0)$ and the ratio between the hot-electron temperature and the lattice temperature $T_{e} / T_{L}$, as a function of the applied field obtained from our Monte Carlo code for a GaAs bulk with $n=10^{16} \mathrm{~cm}^{-3}$ at $T_{L}=300 \mathrm{~K}$. These results are compared with the calculations from the KSBEs (see Fig. 15(b) of Ref.[24]). Our finding for the spin lifetime well agrees with the theoretical results in all the investigated range, while the values of the electron temperature are systematically slightly lower than those obtained from the KSBE approach. Once more time, this small deviation can be due to the fact that the Coulomb scattering is neglected in our computation. In fact, the electron-electron (e-e) scattering is very important in determining the hot-electron temperature and the hotelectron temperature influences both the electron-longitudinal optical (e-LO) phonon and the electron-impurity scattering. Hence the e-e scattering effectively influences the spin relaxation. However, since at high temperatures, the e-e scattering is weak 
compared to e-LO phonon scattering, it leads only to a marginal decrease in the spin relaxation [23]. Moreover, Römer et al. [52] have experimentally shown that, for doping concentrations below the metal-to-insulator transition, the electron-electron interaction is weaker at low impurity densities. In our finding this decrease is surely negligible for the two lower values of donor concentrations, namely $n=10^{13} \mathrm{~cm}^{-3}$ and $n=10^{15} \mathrm{~cm}^{-3}$.

The analysis of the influence on the spin lifetime of the intravalley and intervalley Coulomb scattering at high lattice temperatures is a very interesting problem which deserves further detailed investigations that will be the subject of a forthcoming paper.

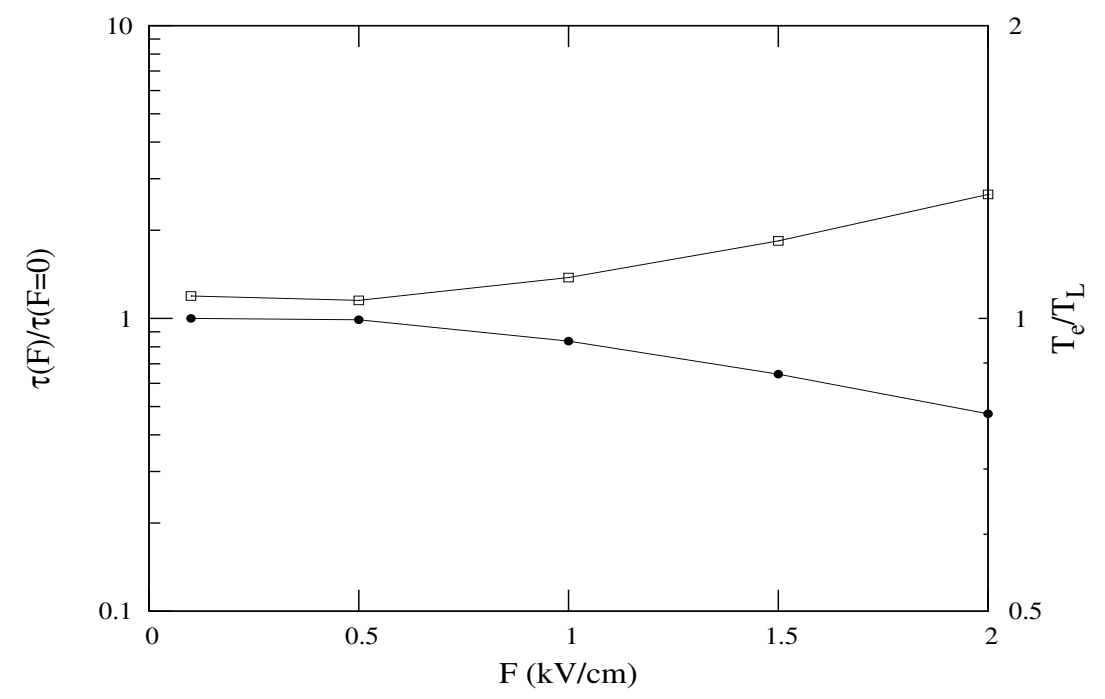

Figure 6. Ratio of the spin relaxation time under electric field to the electric-fieldfree one $\tau(F) / \tau(F=0)$ and ratio between the hot-electron temperature and the lattice temperature $T_{e} / T_{L}$ as a function of the applied field obtained from our Monte Carlo code, $n=10^{16} \mathrm{~cm}^{-3}, T_{L}=300 \mathrm{~K}$.

\section{Conclusions}

A full understanding of the role played by the lattice temperature, the doping density and the amplitude of high-intensity electric field on the electron spin dynamics in semiconductors is essential for the design and the fabrication of spintronic devices.

In this work we have studied the spin lifetimes of an ensemble of conduction electrons, drifting in doped n-type GaAs bulk crystal, by using a semiclassical Monte Carlo transport model. The spin depolarization is investigated in a wide range of lattice temperatures and doping densities by simulating the electrons driven by an electric field with amplitude $0.1<F<6 \mathrm{kV} / \mathrm{cm}$, including both the $\Gamma$-valley and the $L$-valleys into the spin dephasing dynamics.

Our results show that the electron spin lifetime is not marginally influenced by the driving electric field, the lattice temperature and the impurity density, which hence represent key parameters into the depolarization process. We find a significant reduction of the average spin polarization lifetime at high values of the electric field, caused by the 
stronger spin-orbit coupling of electrons in the $L$-valleys. In the nondegenerate regime the doping density considerably affects spin lifetimes at nitrogenum temperature and

weak intensity of the driving electric field. Moreover, for field amplitudes greater than $2.5 \mathrm{kV} / \mathrm{cm}$, we observe spin lifetimes longer at room lattice temperatures with respect to those observed at $T_{L}=77 \mathrm{~K}$.

The prediction capability of our numerical code has been positively validated through a comparison with the available experimental results and with the calculations obtained by a different theoretical approach.

\section{Acknowledgments}

This work was partially supported by MIUR and CNISM-INFM. The authors acknowledge CASPUR for the computing support via the standard HPC grant 2010. Authors are very thankful to Prof. Dr. M.W. Wu, who improved this work by precious comments and suggestions.

\section{References}

[1] Fabian J and Das Sarma S 1999 J. Vac. Sci. Technol. B 17, 1708

[2] Wolf S A, Awschalom D D, Buhrman R A, Daughton J M, Von Molnár S, Roukes M L, Chtchelkanova A Y and Treger D M 2001 Science 294, 1488

[3] Awschalom D D and Flatté M E 2007 Nature Phys. 3, 153

[4] Fabian J, Matos-Abiage A, Ertler C, Stano P and Žutić I 2007 Acta Phys. Slov. 57, 565

[5] Lou X, Adelmann C, Crooker S A, Garlid E S, Zhang J, Madhukar Reddy K S, Flexner S D, Palmstrøm C J and Crowell P A 2007 Nature Phys. 3, 197

[6] Jonker B T, Kioseoglou G, Hanbicki A T, Li C H and Thompson P E 2007 Nature Phys. 3, 542

[7] Dyakonov M I (Ed.) 2008 Spin Physics in Semiconductors (Berlin) Springer, Solid-State Sciences, Vol. 110, and references therein.

[8] Gulayev Y V, Zilberman P E, Panas A I and Epshtein E M 2009 Usp. Fiz. Nauk 52 (4), 335

[9] Flatté M E 2009 Nature 462, 419

[10] Dash S P, Sharma S, Patel R S, De Jong M P and Jansen R 2009 Nature 462, 491

[11] Wu M W, Jiang J H and Weng M Q 2010 Physics Reports, 49361.

[12] Hägele D, Oestreich M, Rühle W W, Nestle N and Eberl K 1998 Appl. Phys. Lett. 73, 1580

[13] Sanada H, Arata I, Ohno Y, Chen Z, Kayanuma K, Oka Y, Matsukura F and Ohno H 2002 Appl. Phys. Lett. 81, 2788

[14] Sato Y, Takahashi Y, Kawamura Y and Kawaguchi H 2004 Jpn. J. Appl. Phys. 43, L230

[15] Stephens J, Berezovsky J, McGuire J P, Sham L J, Gossard A C and Awschalom D D 2004 Phys. Rev. Lett. 93, 097602

[16] Crooker S A and Smith D L 2005 Phys. Rev. Lett. 94, 236601

[17] Furis M, Smith D L, Crooker S A and Reno J L 2006 Appl. Phys. Lett. 89, 102102

[18] Beck M, Metzner C, Malzer S and Döhler G H 2006 Europhys. Lett. 75, 597

[19] Ivchenko E L, Lyanda-Geller Y B and Pikus G E 1990 Sov. Phys.-JEPT, 71, 550

[20] Wu M W and Ning C Z 2000 Phys. Status Solidi B 222, 523

[21] Weng M Q and Wu M W 2003 J. Appl. Phys. 93, 410

[22] Weng M Q, Wu M W and Jiang L 2004 Phys. Rew. B 69, 245320

[23] Zhang P, Zhou J and Wu M W 2008 Phys. Rev. B 77, 235323

[24] Jiang J H and Wu M W 2009 Phys. Rev. B 79, 125206

[25] Mishchenko E G and Halperin B I 2003 Phys. Rev. B 68, 045317 
[26] Saikin S 2004 J. Phys.: Condens. Matter 16, 5071

[27] Kiselev A A and Kim K W 2000 Phys. Rev. B 61, 13115

[28] Bournel A, Dollfus P, Cassan E and Hesto P 2000 Appl. Phys. Lett. 77, 2346

[29] Barry E A, Kiselev A A, Kim K W 2003 Appl. Phys. Lett. 82, 3686

[30] Saikin S, Shen M, Cheng M C and Privman V 2003 J. Appl. Phys. 94, 1769

[31] Pramanik S, Bandyopadhyay S and Cahay M 2003 Phys. Rev. B 68, 075313

[32] Shen M, Saikin S, Cheng M C and Privman V 2004 Mathematics and Computers in Simulation 65, 351

[33] Pershin Y 2005 Phys. Rev. B 71, 155317

[34] Saikin S, Shen M and Cheng M C 2006 J. Phys.: Condens. Matter 18, 1535

[35] Yu Z G and Flatté M E 2002 Phys. Rev. B 66, 201202; 2002 Phys. Rev. B 66, 235302

[36] Martin I 2003 Phys. Rev. B 67, 014421

[37] Hruška M, Kos Š, Crooker S A, Saxena A and Smith D L 2006 Phys. Rev. B 73, 075306

[38] Fu J Y, Weng M Q and Wu M W 2008 Physica E 40, 2890

[39] Elliott R J 1954 Phys. Rev. 96, 266

[40] Yafet Y 1963 Solid State Physics, edited by F. Seitz and D. Turnbull (New York) Academic, Vol. 14, p. 2.

[41] Dyakonov M I and Perel V I 1971 Sov. Phys. - Solid State 13, 3023

[42] Dyakonov M I 2006 Physica E 35, 246

[43] Bir G L, Aronov A G and Pikus G E 1976 Sov. Phys. - JETP 42, 705

[44] Abragam A 1961 The Principles of Nuclear Magnetism, (Oxford) Clarendon Press.

[45] Paget D, Lampel G, Sapoval B and Safarov V I 1977 Phys. Rev. B 15, 5780

[46] Pikus G E and Titkov A N 1989 Optical Orientation, edited by Meyer F, (Leningrad) Nauka.

[47] Litvinenko K L, Leontiadou M A, Li J, Clowes S K, Emeny M T, Ashley T, Pidgeon C R, Cohen L F, Murdin B N 2010 Appl. Phys. Lett. 96, 111107

[48] Jacoboni C and Lugli P 1989 The Monte Carlo Method for Semiconductor Device Simulation, edited by S. Selberherr, (Wien) Springer.

[49] Tomizawa K 1993 Numerical simulation of submicron semiconductor devices, (London, Boston) Artech House.

[50] Moglestue C 1993 Monte Carlo Simulation of Semiconductor Devices, (London) Chapman and Hall.

[51] Persano Adorno D, Zarcone M and Ferrante G 2000 Laser Phys. 66, 310

[52] Römer M, Bernien H, Müller G, Schuh D, Hübner J and Oestreich M 2010 Phys. Rev. B 81, 075216

[53] Krich J J and Halperin B I 2007 Phys. Rev. Lett. 98, 226802 